\title{
A Current Status on Diversity and Distribution of Black Flies (Insecta: Diptera:Simuliidae) in India
}

\section{Bulganin Mitra', Sankarsan Roy ${ }^{1 *}$, Souradip

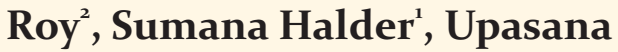 Chattopadhyay ${ }^{3}$, Subhrajit Bhaumik ${ }^{2}$}

${ }^{1}$ Zoological Survey of India, M-Block, New Alipore, Kolkata, India

${ }^{2}$ Post Graduate Department of Zoology, Vidyasagar College, Salt Lake City, Kolkata, India

${ }^{3}$ Post Graduate Department of Zoology, Barasat Government College, Barasat, Kolkata, India

Key words: Bio-geographic zones

\section{Introduction:}

Simuliidae, or black flies, under the infra-order Culicomorpha of the suborder Nematocera are a group of dipteran flies (Pape et al., 2011) which are well known for being an annoyance to the man and animals of different regions. This group of insects are belonged to the aquatic insect communities and also play a role as detrivores in lotic ecosystems (Cummins, 1987). These flies are ideal organisms to understand species richness of aquatic insects. A total of 2219 species under 26 genera of Simuliidae have been reported from the World (Adler \& Crosskey, 2016). Mitra \& Sharma (2010) published a checklist of Indian simuliid where they mentioned a list of 67 species, among which 11 species were mentioned up to genus level. Till date, there is no consolidated list of Simuliidae reported from India. Present communication is a compilation of work based on recent classification and valid nomenclature followed after Systema Dipterorum (Pape and Thomson, 2013) and World Catalogue of Simuliidae (Adler \& Crosskey, 2016) and others available literature along with its distribution in states and union territories of India. Altogether, 69 species belonging to eight subgenera and a single genus reported so far, which includes onlyvalid species reported from India (Table - 1).

\section{History of taxonomic research:}

Probably, Latreille is the first man who described the type genus Simulium in the year 1802. Later, Meigen (1818) made it a well-def ined group and finally the family named Simuliidae was designated with a single genus by Newman (1834). As far knowledge goes, Becher (1885) was the first person who described a species of Simuliidae from India. Afterward, several workers like Brunetti (1911), Senior-

\section{Abstract}

Till date a total of 69 species of eight sub-genera under one genus belonging to the family Simuliidae of the order Diptera are reported from India, which is $3.11 \%$ of the total world extant simuliid fauna. Among these, $30.43 \%$ species are restricted to India. Out of 36 states and union territories of India, simuliids are reported only from 20 states and UTs. Highest numbers of species are reported from the Himalayan biogeographic zone (68.11\%) and lowest in IndoGangetic plains (5.79\%). So far, nothing has been known from Islands biogeographic zone.

White (1922a,b); Edwards (1927); Puri (1932 a,b,c,d,e); Puri (1933 a,b,c) contributed new species in the Indian simuliid fauna. Datta (1973; 1974; 1975; 1985), Datta \& Pal (1975) also studied the Indian simuliid fauna and described several species from this country. In addition to, Datta (1983) reviewed the family Simuliidae of Oriental Region. Recently, Takaoka \& Somboon (2008); Takaoka et al., (2010; 2011); Anbalagan (2014; 2015 a,b) described several species of Simuliidae from India. Several new distributional records of Indian simuliids have been reported by Datta $(1978 ; 1985 ; 1992 ; 1997)$, Henry et al. (2011), Shrestha \& Takaoka (2009); Mitra et al. (2006); DuttaSaha et al. (2012).

\section{Distinguishing characters:}

Adults are usually small, dark coloured flies with a short horn-like antenna (generally 11-segmented) and short mouthparts. Mandibles and maxilla are usually armed with teeth and adapted for blood sucking. Thorax is remarkably arched. Wings are broad, three anterior veins are more prominent than the posterior vein. The mature larva is more or less $6 \mathrm{~mm}$ long, cylindrical and swollen posteriorly. There is a pair of dark spots laterally and a proleg ventrally in the thoracic region. (Datta, 1980)

\section{Habit and habitats:}

The members of this family were found most abundantly in hilly terrain with watercourses like water channels, streams, nearby areas of the river etc. Actually, these areas are most common for their breeding (Datta, 1980). Females usually lay eggs in the vegetation (whatever it may live or dead), rocks or other suitable submerged substrate (Datta et al., 1975). After emerging from the pupae they

*Corresponding Author: sroy.zoology@gmail.com 
usually used to fly near the water bodies. Most of the species of the simuliid flies appear to mate in the mid-air and sheltered near the bushes and tree tops. (Datta, 1980). These flies are with sucking type of mouthparts as these are adapted to blood sucking of several animals and human.

\section{Biology and economic importance:}

Black flies are one of the most common and important groups of insects with medical and economic importance. Black flies have the symbiotic relations with the fungi of the class Trichomycetes, and common in the larvae as the larvae also shows symbiotic relations. Even in Africa, many larvae show phoratic relations with small crabs. These worms (which) can be seen in the fly's body appearing like a loop (Upton, 2005; Adler, 2003; Azevedo, 2006). The simuliids are economically important as the females of most of the species are haematophagous and their habit of blood-feeding is closely related to the transmission of certain diseases like human and nonhuman onchocerciasis, leucocytozoonosis, avian trypanosomiasis, rabbit myxomatosis in different parts of the world (Fallis, 1964). Though in India, several records of biting of black flies were reported (Lewis, 1974; Datta \& Dasgupta, 1975) but there was no direct evidence of disease transmission (Datta, 1980). Itching and localized swelling and inflammation are very common responses that occur at the site of a bite.

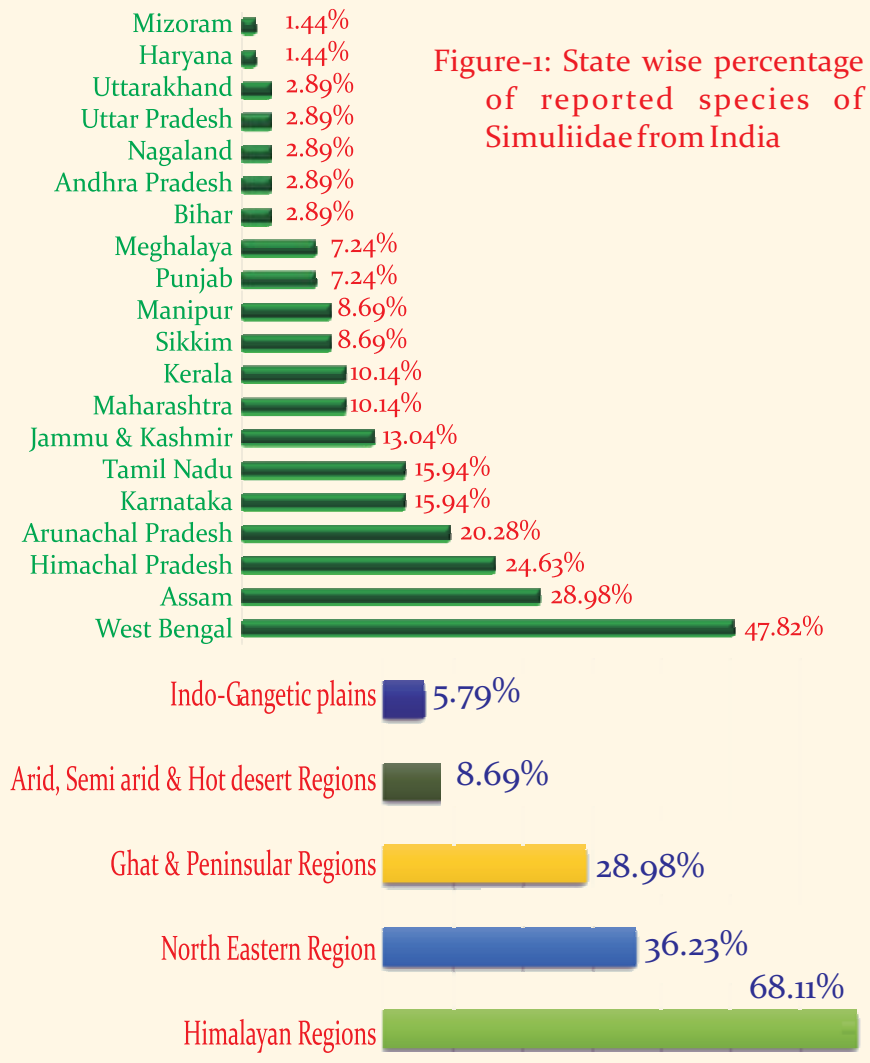

Figure-2: Percentage in different Bio-geographic zones of India
Table-1: Distribution of species in different states and biogeographic zones of India

[Biogeographical zones are classified as Alfred et al., 2001]

Sl. Name of the species

Distribution Distribution outside in India India

B.Z.India*

1. Simulium (Eusimulium) aureum Fries, 1824 TL: Sweden. Scania: Esperod \& Bjornstorp

Jammu \&
Kashmir,
Himachal
Pradesh
HR

Eurasia, Iceland, China, Sweden, Austria, Latvia, Belarus, Belgium, Italy, Britain, Czech Republic, Denmark,Estonia, Japan, Finland, France, Pakistan, Germany, Iceland, Ireland, Lithuania, Russia, Norway, Romania Netherlands, Poland, Siberia, Slovakia, Spain, Sweden, Switzerland, Ukraine, Bulgaria, Uzbekistan, Kazakhstan,

2. Simulium (Eusimulium) weiningense Chen \& Guizhou, China

Zhang, 1997; TL: China. Guizhou: Weining

3. Simulium (Gomphostilbia) Assam NER Nepal bucolicum Datta, 1975; TL: Sonai Rupai Forest

4. Simulium (Gomphostilbia) Assam NER Restricted within India fidum Datta, 1975; TL: Assam: Sonai Rupai Forest

5. Simulium (Gomphostilbia) Assam NER Restricted within India litoreum Datta, 1975; TL: Assam: Sonai Rupai Forest

6. Simulium (Gomphostilbia) Tamil Nadu China, Sri Lanka pattoni Senior-white, 1922 GPR

TL: Madras: Coonoor (HT A BMNH)

7. Simulium (Gomphostilbia) Assam NER Restricted within India unum Datta, 1975; TL: Assam: Sonai Rupai Forest

8. Simulium (Gomphostilbia) darjeelingense Datta, 1973; TL: W. Bengal, Lebong

W. Bengal, Nepal

Arunachal P.,

Assam.

HR, NER

9. Simulium (Gomphostilbia) metatarsale Brunetti, 1911 TL: Kurseong, E.Himalaya

10 Simulium (Gomphostilbia) tenuistylum Datta, 1973; TL: W.Bengal, Lebong

W. Bengal, Sikkim HR

China, Taiwan [West Malaysia, Indonesia W. Bengal, Restricted within India Sikkim,

Assam, Arunachal P. HR, NER

11. Simulium (Montisimulium) W. Bengal China (Sichuan) dasguptai Datta, 1974; $\quad$ HR TL: W. Bengal, Darjeeling

12. Simulium (Montisimulium) West Bengal Bhutan, Nepal dattai Takaoka \& Somboon, HR 2008; TL: W. Bengal

13. Simulium (Montisimulium) West Bengal China (Tibet) ghoomense Dutta, 1973; TL: HR India, W.Bengal, Darjeeling

14. Simulium (Montisimulium) West Bengal, Bhutan, China (Tibet), nemorivagum Datta, 1973 Arunachal P. Nepal, Pakistan TL: W.Bengal, Darjeeling HR, NER

15. Simulium (Montisimulium) West Bengal China (Henan) yuntaiense Chen, Wen \& HR Wei, 2006; TL: China 


\section{REVIEW ARTICLE}

16. Simulium (Nevermannia) praelargum Datta, 1973; TL: W.Bengal, Darjeeling

17. Simulium (Nevermannia) rufithorax Brunetti, 1911; TL: W.Bengal, Darjeeling, Kurseong

18. Simulium (Nevermannia) senile Brunetti, 1911; TL: Simla: Phagu

19. Simulium (Nevermannia) aureohirtum Brunetti, 1911 TL: Umling, Meghalaya

20. Simulium (Nevermannia) gracilis Datta, 1973;

TL: W.Bengal, Darjeeling

21. Simulium (Nevermannia) puri Datta, 1973; TL: W.Bengal, Darjeeling

22 Simulium (Tetisimulium) stevensoni Edwards, 1927 TL: Jammu \& Kashmir: Chillas, Gilgit(LTA BMNH)

23. Simulium (Simulium) ephemerophilum Rubstov, 1947; TL: Tajikistan. Hissar

24. Simulium (Simulium) rashidi Lewis, 1973; TL: Pakistan. Bahrain (HT A BMNH)

25. Simulium (Simulium) gravelyi Puri, 1933; TL: India. Madras: Kodaikanal, Palmi Hills, Law's Ghat, Silver Cascades (4ABMNH)

26. Simulium (Simulium) griseifrons Brunetti, 1911; TL: Uttar P.: Kumaon, Kalighat (Uttarakhand)

27. Simulium (Himalayum ) indicum Becher 1885; TL: Assam

28. Simulium (Simulium) tenuitarsus Puri, 1933 TL: West Bengal: Bengal, Terai, Marianbarie
West Bengal China (Tibet), Nepal HR

Karnataka, Restricted within India Maharashtra,

W. Bengal

HR, GPR

Himachal Restricted within India

Pradesh

HR

Assam, Nepal, Pakistan,

Arunachal P. Philippines(Bohol, Luzon,

Himachal P. Mindanao, Negros,

Karnataka, Palawan,Panay),Sri Lanka

Maharashtra Taiwan, Thailand, Guam,

Meghalaya, Vietnam, Bhutan, Borneo

Manipur, China (Fu,Gd,Gu,Hainan,

Mizoram, Macao, HK, Si, Tibet, Yu),

Tamil Nadu, Indonesia (Flores, Java,

West Bengal, Kalimantan, Sulawesi,

Haryana NE, Maluku,Sumatra), Japan

GPR, HR, (Hon, Ky, NI,Sh), Malaysia

ASHD (Malaya, Sabah,Sarawak)

West Bengal China (Tibet),

HR Nepal

West Bengal China (Tibet),

HR Nepal

Jammu and Pakistan

Kashmir

HR

Himachal P. Pakistan, Central Asia,

HR Tajikistan, Uzbekistan, Kyrgyzsthan, Pakistan

Himachal P. Pakistan

HR

Tamil Nadu China (Tibet)

GPR

Himachal P., Malaya, Thailand,

Jammu \& China (Tibet), Pakistan

Kashmir,

Uttarakhand

HR

Assam, Afghanistan, Myanmar,

Arunachal P., Bangladesh, Bhutan,

Himachal P., China (Tibet), Myanmar,

Jammu \& Nepal, Pakistan (Jammu

Kashmir, \& Kashmir), Siam,

Karnataka, Thailand

Maharashtra,

Manipur,

Meghalaya,

Nagaland,

Sikkim, Uttar

P., W. Bengal.

NER, HR,

GPR, IGP

West Bengal Restricted within India HR
29. Simulium (Simulium) hirtipannus Puri, 1932 TL: Assam: Manipur , Imphal

30. Simulium (Simulium) barraudi Puri, 1932; TL: India. Jammu \& Kashmir: Prang \& Nara-Nag (ST A BMNH)

31. Simulium (Simulium) dentatum Puri, 1932;

TL: W.Bengal: Darjeeling, Kurseong \& Tarai, Marianbarie (2A BMNH)

32. Simulium (Simulium) digitatum Puri, 1932;

TL: Himachal Pradesh:

Chhota Simla \&

Chadwick Falls; Punjab:

Kasauli \& Dagshai

33. Simulium (Simulium) lineothorax Puri, 1932; TL: Assam: Cachar, Haflong (2 A BMNH)

34. Simulium (Simulium) novolineatum Puri, 1933; TL: W. Bengal: Terai Bengal, Marianbarie

35. Simulium (Simulium) asishi Datta, 1985. TL: Jalpaiguri, Terai

36. Simulium (Simulium) nodosum Puri, 1933;

TL: Mysore: Coorg, Cauvery River, Frazerpet

37. Simulium (Simulium) adventicium Datta, 1985; TL: Shalimar, Srinagar, Jammu \& Kashmir

38. Simulium (Simulium) consimile Puri, 1932 TL: Punjab: Ambala, Chandigarh (2A BMNH)

39. Simulium (Simulium) grisescens Brunetti, 1911; TL: W.Bengal: Darjeeling, Kurseong.

40. Simulium (Simulium) kapuri Datta, 1975; TL: Assam:Sonai Rupai Forest

41. Simulium (Simulium) pallidum Puri, 1932; TL: Punjab: Kasauli (5A BMNH)

42. Simulium (Simulium) palmatum Puri, 1932;
Manipur, China (Fu, Gu, Tibet)

Assam,

W. Bengal

NER, HR

Himachal P., China (Tibet), Pakistan

Jammu \&

Kashmir

HR

Assam, Bhutan, China (Tibet)

Meghalaya,

Sikkim

W. Bengal

NER, HR

Himachal P., China (Gd, Tibet)

Punjab

HR, ASHD

Assam, Restricted within India

Tamil Nadu

NER, GPR

Arunachal P., Burma

Assam, Uttar

Karnataka,

W. Bengal,

Uttarakhand

NER, IGP,

GPR, HR

W. Bengal Restricted within India

HR

Karnataka, China (Fu, Hainan, HK, Assam, W. Tibet, Yu), Thailand,

Bengal,NER, Vietnam

GPR,HR

Himachal P., Restricted within India

Jammu \&

Kashmir

HR

Himachal P., Pakistan, Sri Lanka

Punjab

HR, ASHD

W. Bengal, Bhutan, Pakistan, Arunachal P., Taiwan

Assam, Kerala,

Himachal P.

Karnataka,

Maharashtra,

Meghalaya,

Manipur,

Nagaland,

Punjab,

Tamil Nadu.

NER, HR,

GPR,ASHD

Assam,

W. Bengal

NER, HR

Punjab, Pakistan

Himachal P.,

ASHD, HR

Maharashtra, Restricted within India

Karnataka, 
TL: Maharashtra: Bombay, Kerala Savantvadi (4 A BMNH) GRP

43. Simulium (Simulium) striatum Brunetti, 1912; TL: Sri Lanka:Peradeniya

Karnataka, Sri Lanka, Bhutan

Kerala,Assam

Maharashtra,

Tamil nadu,

GPR, NER

44. Simulium (Simulium) biforaminiferum Datta, 1974; TL: W. Bengal, Darjeeling

45. Simulium (Simulium) nigrifacies Datta, 1974; TL: W.Bengal, Darjeeling

46. Simulium (Simulium) nitidithorax Puri, 1932; TL: India, W. Bengal: Terai, Marianbarie (3 A BMNH)

47. Simulium (Simulium) ramosum Puri, 1932; TL: Simla (2A BMNH)

48. Simulium (Simulium) rufibasis Brunetti, 1911; TL: Kurseong, Darjeeling

49. Simulium (Simulium) gurneyae Senior-White, 1922; TL: Madras: Coonoor (HT A BMNH)

50. Simulium (Simulium) himalayense Puri, 1932; TL: Simla (8 A BMNH)

51. Simulium (Simulium) nilgiricum Puri, 1932; TL: Madras: Coonoor, (1 A BMNH)

52. Simulium (Simulium) christophersi Puri, 1932; TL: Jammu \& Kashmir: Nara-Nag (4 A BMNH)

53. Simulium (Simulium) howletti Puri, 1932; TL: Bihar: Pusa

54. Simulium (Simulium) palniense Puri, 1932; TL: Madras:Kodaikanal (5A BMNH)

55. Simulium (Simulium) singtamense Datta \& Pal, 1975; TL: W.Bengal: Darjeeling,

56. Simulium (Wilhelmia) pseudequinum Seguy, 1921; TL: Canary Is. Punjab
W. Bengal Bhutan HR

Karnataka, China (Tibet)

Kerala,

Andhra P.,

Tamil nadu,

W. Bengal

GPR, HR

Arunachal P., China (Fu, Hainan,

W. Bengal Tibet), Malaysia

NER, HR

Himachal P., Nepal, Pakistan, Bhutan, W. Bengal Thailand

HR

Arunachal P., China, Japan, Korea

Himachal P., (South), Ryukyu islands,

Meghalaya, Nepal, Pakistan, Taiwan,

Manipur, Thailand, Vietnam

Sikkim, W.

Bengal, Assam

NER, HR

Karnataka, Restricted within India

Maharashtra,

Tamil Nadu

GPR

Arunachal P., China (Tibet), Nepal,

Assam, Bihar Pakistan

Himachal P.,

Manipur,

Sikkim, W.

Bengal. NER,

HR, IGP

Tamil Nadu Sri Lanka

GPR

Himachal P., China (Tibet)

Jammu \&

Kashmir,

Assam,

Arunachal P.

HR, NER

Bihar China (Tibet)

IGP

GPR

W.Bengal Nepal

HR

Jammu \& Canary Islands (Gran

Kashmir, Canaria, Gomera,

HR, ASHD Tenerife),Algeria, Austria, Armenia, Iran,
Tamil Nadu NSL
Cyprus, France, Greece, Britain, China (Sx, Xi)

raq, Azerbaijan, Bosnia,

Georgia,Israel,Italy (incl. Sardinia, Sicily),Jordan, Kazakhstan, Kyrgyzstan, Lebanon, Libya, Serbia, Macedonia, Morocco, Pakistan, Spain, Portugal, Romania, Turkey, Russia (Caucasus),Slovakia, Slovenia,Tajikistan, Tunisia, Turkmenistan, Ukraine (incl. Crimea), Uzbekistan,Czech Repub.

57. Simulium (Gomphostilbia) Arunachal P., Malaysia, Thailand decuplum Takaoka \& Davies, l995; TL: Malaysia Malaya (HT BMNH)

58. Simulium (Gomphostilbia) Assam parahiyangum Takaoka \& NER Sigit, 1992; TL: Indonesia

59. Simulium(Gomphostilbia) peteri Anbalagan, 2014; TL: Kulathupuzha, Kerala

6o. Simulium (Gomphostilbia) takaokai Anbalagan, 2014; ; GPR

61. Simulium (Gomphostilbia) HR sachini Takaoka \& Henry, 2010; TL: Darjeeling, W.B.

62. Simulium (Gomphostilbia) williei Takaoka \& Thapa, 2010; TL: Darjeeling, W.B.

63. Simulium (Gomphostilbia) Arunachal P., Thailand barnesi Takaoka \& Suzuki, NER 1984; TL: Thailand (HT A BMNH)

64. Simulium (Gomphostilbia) Karnataka Restricted within India cauveryense Anbalagan, 2015; TL: Kushalanagar, Kodagu, Karnataka

65. Simulium subratai Takaoka, Thapa \& Henry, 2011; TL: India Darjeeling: Dali, (HT F PG College)

66. Simulium (Gomphostilbia) Keral kottoorense Anbalagan, 2015; TL: Kot-toor, Tiruvananthapuram, Kerala

67 Simulium (Gomphostilbia) panagudiense Anbalagan, 2015; TL: Panagudi, Tirunelveli Tamil Nadu

68 Simulium (Simulium) alajense Rubtsov, 1938; TL: Tajikistan \& Kirgizia

69 Simulium (Simulium) pradyai Takaoka \& Somboon, 2008; TL: Bhutan

Abbreviation used: TL: Type Locality, HR: Himalayan Region; IGP: Indo Gangetic Plains; GPR: Ghats \& peninsular regions; ASHD: Arid, SemiArid \& Hot desert region; NER: North Eastern region; NSL: No specific Locality, IS: Islands, P.: Pradesh 


\section{REVIEW ARTICLE}

\section{Discussion:}

The present communication provides a list of 69 species (3.11\% of total world simuliid fauna) belonging to eight sub-genera and a single genus in comparison to the total number of species of Simuliidae (2219 species) found worldwide. As per the present data the simuliids are recorded from 20 out of 36 states of India. Of them, West Bengal represents maximum number of Simuliidae about (47.82\%), followed by Assam (28.98\%), Himachal Pradesh (24.63\%), Arunachal Pradesh (20.28\%), Karnataka (15.94\%) and Tamil Nadu (15.94\%), Jammu \& Kashmir (13.04\%), Kerala and Maharashtra (each with 10.14\%), Manipur and Sikkim (each with 8.69\%), Punjab and Meghalaya (each with 7.24\%). Bihar, Uttar Pradesh, Uttarakhand, Nagaland and Andhra Pradesh (each with $2.89 \%)$. Besides this, Haryana and Mizoram each shared $1.44 \%$ of the Simuliidae of India (Table 1, Fig. 1). Out of these 69 simuliid species, 20 species have restricted their distribution within India. Among the six major biogeographic zones of India (Alfred et al., 2001), the maximum number of species $(68.11 \%)$ are reported from the Himalayan regions, followed by North-eastern Regions ( $36.23 \%$ species), Ghats \& Peninsular regions (28.98\% species), Arid-Semiarid \& hot desert regions (8.69\% species), Indo-gangetic plains $(5.79 \%$ species). Nothing has been known from Islands biogeographical zone (Fig.-2). Present document aims to generate up to date distribution data of Indian simuliids. The number of simuliid species in West Bengal is maximum due to extensive survey in this state that has been made by the scientists of ZSI, Kolkata. On the other hand, under explored states are having less number of species. Similarly, more species of this family were reported from the Himalayan region due to more survey and explorations have been made in Himalayan region by both the British taxonomists and Indians as well. Therefore, it is an urgent need to explore every corners of India which may lead discoveries of several new species/new records.

\section{References:}

Adler, P. (2003): Prevalence of the trichomycete fungus Harpellamelusinae (Harpellales: Harpellaceae) in larval black flies (Diptera: Simuliidae) across a heterogeneous environment. Mycologia , 95(4):577-583.

Adler, P.H. \& Crosskey, R.W. (2016): World blackflies (Diptera: Simuliidae) : A comprehensive revision of the taxonomic and geographical inventory. BioMIA. Pub. by: Clemson University, South Carolina.

Alfred, J.R.B., Das A.K. \& Sanyal A.K. (2001): In: Ecosystems of India. ENVIS Centre, ZSI, Kolkata, pp. 1-33, 93-122, 317-410.

Anbalagan, S., Prasanna, V.A., Kannan, M., Dinakaran, S. \& Krishnan, M. (2014): Two new species of Simulium (Gomphostilbia) (Diptera: Simuliidae) from Peninsular India with keys to Peninsular Indian members of the genus Simulium. Zootaxa, 3861(5):451-465

Anbalagan, S., Prasanna, V.A., Kannan, M., Dinakaran, S. \&
Ambient Science, 2017: Vol. 04(1); 21-26 DOI:10.21276/ambi.2017.04.1.rv01

Krishnan, M. (2015a): Simulium (Gomphostilbia) (Diptera: Simuliidae) from Southern Western Ghats, India: Two new species and DNA barcoding. Acta Trop., 149: 94-105.

Anbalagan, S., Balachandran, C., Prasanna, V.A., Kannan, M., Dinakaran, S. \& Krishnan, M. (2015b): A new species of Simulium (Gomphostilbia) (Diptera: Simuliidae) from South India, with keys to Indian members of the subgenus Gomphostilbia. Zootaxa, 3974(4):555-563.

Azevedo, L. (2006). Spatial and Temporal Distribution of Blackflies (Diptera: Simuliidae) in the Itatiaia National Park, Brazil. Neotrop. Entomol., 35(4):542-550.

Becher, E. (1885): A new species of Simulium from Assam. J. Asiatic Soc. Bengal, 53 (2): 199-200.

Brunetti, E. (1911): New Oriental Nemocera. Rec. Indian Mus., 4: 259-316.

Cummins, K.W. (1987): The functional role of black flies in stream ecosystems. In: Kim KC, Merritt RW (eds.). Black Flies: Ecology, population management and annotated world list. Pub. by: The Pennsylvania State University Press.

Datta, M. (1973): New species of black flies (Diptera: Simuliidae) of the subgenera Eusimulium Roubaud and Gomphostilbia Enderlein from the Darjeeling area, India. Orient. Insects, 7: 363-402.

Datta, M. (1974a): Some black flies (Diptera: Simuliidae) of the subgenus Simulium Latreille (s. str.) from the Darjeeling area (India). Orient. Insects, 8:15-27.

Datta, M. (1974b): New species of black flies (Diptera: Simuliidae) from the Darjeeling area, India. Orient. Insects, 8: 457-468.

Datta, M. (1975): Simuliidae (Diptera) from Assam foot-hills, India. Japanese Journal of Sanitary Zoology, 26:31-40.

Datta, M. (1978): Simuliidae (Diptera) from Sikkim, India. Bull. Zool. Sur. India, 1(3):253-256.

Datta, M. (1980): Collection and preservation of blackflies (Diptera :Simuliidae). Proc. Workshop Tech. Parasitol. Zool. Surv. India: $75-82$.

Datta, M. (1983): A review of Simuliidae (Diptera) from the Oriental Region. Orient. Insects, 17(1):215-267.

Datta, M. (1985): Notes on Black flies (Diptera: Simuliidae) from Kashmir, India. Bull. Zool. Sur. India, 7(2-3):231-236.

Datta, M. (1992): An overview of the Simuliidae (Diptera) of West Bengal, India. L. Bengal Nat. Hist. Soc., 11: 41-62.

Datta, M. (1997): Insecta: Diptera: Simuliidae. In: Zoological Survey of India, Fauna of West Bengal, State fauna series, 3(7):127-162.

Datta, M. \& Dasgupta, B. (1972): On the collection and rearing of black flies (Simuliidae: Diptera) of Darjiling. Indian Biol., 4: 54-59.

Datta, M. \& Pal, T.K. (1975): Few rare black fly species (Diptera :Simuliidae) of the subgenus Simulium Latreille (s. str.) from the Darjiling area, India. Proc. Indian Acad. Sci. (B), 81:154-161.

Datta, M., Dey, R.K., Pal, A.K. \& Pal, T.K. (1975): Ecology of the black flies in (Diptera: Simuliidae) in Darjeeling area. Proc. Indian Acad. Sci. (B), 81(1): 7-19.

Dutta Saha, P., Sharma, R.M. \&Mitra, B. (2012): Insecta: Diptera. In: Zoological Survey of India, Fauna of Maharashtra, State fauna Series, 20: 531-538.

Edwards, F.W. (1927): Two new Simulium from Kashmir. Bull. Ent. 
Res., 18:169-170.

Fallis, A.M. (1964): Feeding and related behaviour of female Simuliidae (Diptera). Exper. Parasitol., 15: 439-470.

Henry, W., Thapa, S., Adler, P.H., Dey, S.K. \& Varma, R. (2011): Cytotaxonomy of Simulium (Montisimulium) ghoomense (Diptera: Simuliidae) from the Darjeeling Hills, India. Zootaxa, 2872: 49-57

Latreille, P.A. (1802): Histoire naturelle, generaleetparticuliere, des crustaces et des insectes. Tome troisieme. Dufart, Paris. $\mathrm{xii}+13-467+1 \mathrm{pp}$.

Lewis, D.J. (1974): Man-biting Simuliidae (Diptera) of north India. Israel J. Entomol., 9:23-53.

Meigen, J.W. (1818): Systematische Beschreibung der bekannten europäischen zweiflügeligen Insekten. Erster Teil FW Forstmann, Aachen, xxxvi + 332 pp; Pinder LCV.

Mitra, B., Lahiri, A.R. \& Mukherjee, M. (2006): Insecta: Diptera: Nematocera. In: Zoological Survey of India, Fauna of Arunachal Pradesh, State fauna Series, 13(2): 225-255.

Mitra B. \& Sharma R.M. (2010): Checklist of Indian Black flies (Insecta: Diptera: Simuliidae). Zoological survey of India (www.zsi.gov.in/checklist)

Newman, E. (1834): Attempted division of British insects into natural orders. Ent. Mag., 2:379-431.

Pape, T., Blagoderov V. \& Mostovski M.B. (2011): Order DIPTERA Linnaeus, 1758. In: Zhang, Z.-Q. (Ed.) Animal biodiversity: An outline of higher-level classification and survey of taxonomic richness. Zootaxa, 3148:222-229.

Pape, T. \& Evenhuis N.L. (eds.)(2013): SystemaDipterorum, Version-1.5.67 records. http://www.diptera.org/

Puri, I.M. (1932a): Studies on Indian Simuliidae. Part-I. Simuliumhimalayense sp. n.; Simuliumgurneyae SeniorWhite; and Simuliumnilgiricum sp. n. Indian J. Med. Res., 19: $883-898$.

Puri, I.M. (1932b): Studies on Indian Simuliidae. Part-II. Description of males, females and pupae of Simuliumrufibasis Brunetti, its variety fasciatumnov. var. and of three new species from the Himalayas. Indian J. Med. Res., 19: 899-913.

Puri, I.M. (1932C): Studies on Indian Simuliidae. Part-III. Description of males, females and pupae of $S$. griseifrons Brunetti (1911) and of four new species with striped thorax. Indian J. Med. Res., 19:1125-1143.
Puri, I.M. (1932d): On Indian Simuliidae. Part IV. Descriptions of two new species from north-east India, Simuliumhowletti sp. $\mathrm{n}$. and Simuliumhirtipennis sp. n., with a note on $S$. ornatum Meigen. Indian J. med. Res., 20: 505-514.

Puri, I.M. (1932e): Studies on Indian Simuliidae. Part-V. Species and varieties of the striatum series. Indian J. Med. Res., 20: 515-532.

Puri I.M. (1933a): Studies on Indian Simuliidae. Part VI. Descriptions of males, females and pupae of two new species from Palni Hills and of male and pupa of $S$. tenuitarsus sp.n. from Bengal Terai. Indian J. Medi. Res., 20(3):803-812.

Puri, I.M. (1933b): Studies on Indian Simuliidae. Part VII. Description of larvae, pupa and females of Simuliumnodosum sp. nov., with an appendix dealing with $S$. novolineatum. nov. nom. (=S. lineatumPuri). Indian J. Med. Res., 20:813-817.

Puri, I.M. (1933c): Studies on Indian Simuliidae. Part VIII. Description of larvae, pupa, male \& females of $S$. aureohirtum Brunetti and S. aureumflies. Indian J. Med. Res., 21:1-10.

Senior-White R.A. (1922a): Notes on Indian Diptera. 1. Diptera from the Khasia Hills. 2. Tabanidae in the collection of the forest zoologist. 3. New species of Diptera from the Indian Region. Mem. Dept. Agricul. India, Entom. Ser., 7: 83-170.

Senior-White, R.A. (1922b): New species of Diptera from the Indian Region. Mem. Dept. Agricul. India, Entom. Ser., 7: 107169, pls 11-15.

Shrestha, S. \& Takaoka, H. (2009): New records of ten species of Black flies (Diptera: Simuliidae) from Nepal. Lapan Soc. Med. Entomol. Zool., 60 (4):253-258.

Takaoka, H. \& Somboon, P. (2008): Eleven new species and one new record of black flies (Diptera: Simuliidae) from Bhutan. Med. Entomol. Zool., 59:213-262.

Takaoka, H., S. Thapa, and W. Henry. (2010): Description of two new species of Simulium (Gomphostilbia) (Diptera: Simuliidae) from Darjeeling, India. Med. Entomol. Zool., 61: 105-110.

Takaoka, H., Thapa, S. \& Henry, W. (2011): A new species of black fly (Diptera: Simuliidae) with an entirely yellow thorax from Darjeeling, India. Zootaxa, 2824: 62-68.

Upton, S. (2005): "TOPIC 45. Order: Diptera; suborder: Nematocera" (On-line). Animal Parasitology Course Lecture Notes. www.kstate.edu/parasitology/classes/625arth45.html. 